\title{
Resistive transitions and the origin of the $n$ value in superconductors with a Gaussian critical-current distribution
}

\author{
H. S. Edelman and D. C. Larbalestier ${ }^{\mathrm{a})}$ \\ Applied Superconductivity Center and Department of Physics, University of Wisconsin-Madison, Madison, \\ Wisconsin 53706
}

(Received February 24, 1993; accepted for publication 10 May 1993)

\begin{abstract}
The resistive transition of a superconductor with a Gaussian distribution of critical currents is analyzed and exact relations for the voltage-current characteristic and the resistive transition index ( $n$ value) in such a superconductor are developed. Excellent fits are found to the experimental transitions in two very different types of superconductor; a $\mathrm{Nb}$-Ti monofilament and a $\mathrm{Bi}_{2} \mathrm{Sr}_{2} \mathrm{Ca}_{2} \mathrm{Cu}_{3} \mathrm{O}_{10}$ tape.
\end{abstract}

\section{INTRODUCTION}

The curvature of the voltage-current $(V-I)$ characteristic of superconductors in principle contains much information about the physical processes controlling the critical current. Multiple processes can simultaneously affect the transition. One process involves the influence of a distribution of critical currents on the $V-I$ characteristic. Jones, Rhoderick, and Rose-Innes ${ }^{1}$ and Baixeras and Fournet ${ }^{2}$ modeled this process and the formalism of Baixeras and Fournet was later used by Warnes and Larbalestier ${ }^{3,4}$ to detect and control defects within multifilamentary superconductors which limited their overall critical current density. Plummer and Evetts ${ }^{5}$ described the $V-I$ curve that rcsults from a Gaussian $I_{C}$ distribution, and Hampshirc and Jones ${ }^{6}$ divided the $V-I$ characteristic for this special case into three regions and proposed limiting forms for $V(I)$ in each region. More recent articles have analyzed $V-I$ curvature that results entirely from flux creep, ${ }^{7-9}$ or that results from treating polycrystalline superconductors as two-dimensional arrays of Josephson-coupled grains. ${ }^{10}$ There is great interest in determining the principal mechanism that controls the $V$-I curve for high-temperature superconductors in which the flux dynamics are particularly complex. Flux creep generates $V-I$ curvature by introducing uncertainty into the definition of $J_{c}$ at each point within a sample. Critical-current inhomogeneity causes similar curvature even for a sample in which $J_{C}$ can be precisely defined everywhere. While models of these different processes yield results that are quite distinct mathmatically, most predict the same general shape for the $V-I$ curve. The purpose of this article is to derive an exact expression for $V(I)$ which applies at any $I$ to a sample with a Gaussian distribution of well-defined critical currents. This expression removes an important physical implausibility inherent in prior expressions. We successfully fit the expression to data on two very different types of superconducting composite, a $\mathrm{Cu}$-clad $\mathrm{Nb}-\mathrm{Ti}$ monofilament and a Ag-sheathed $\mathrm{Bi}_{2} \mathrm{Sr}_{2} \mathrm{Ca}_{2} \mathrm{Cu}_{3} \mathrm{O}_{10}$ (2223) tape. The expression leads naturally to a formula for the $n$-value, which is widely used to characterize the sharpness of a resistive transition.

${ }^{a}$ Also with the Department of Materials Science and Engineering.

\section{THE MODEL}

The model can be understood by supposing we pass current through a superconducting wire in which $J_{C}$ is constant and well defined at any particular cross section normal to the current direction. The critical current at position $x$ is then $I_{C}(x)=A(x) J_{C}(x)$. Distributions of $I_{C}(x)$ may arise from variations in the cross-sectional area $A$ due to, for example, sausaging, cracks, or surface defects; or from variations in $J_{C}$ due to, for example, composition, defect density, or grain size. If many factors vary independently, the central limit theorem predicts that the resulting $I_{C}(x)$ will approach a Gaussian distribution. Such a model has been previously discussed, but earlier treatments did not adequately account for the important fact that a Gaussian distribution can extend to unphysical negative currents. Since none of the above factors can suppress $I_{C}(x)$ below zero, their variation is not truly independent and the negative tail of the Gaussian distribution contracts into a delta function at $I_{C}=0$, as shown in Fig. 1.

Following Baixeras and Fournet, we write the voltage along the wire carrying a current $I$ as

$$
V(I)=R \int_{0}^{I} P(i)(I-i) d i,
$$

where $R$ is the normal-state resistance, usually determined by a resistive shunt, and $P(i) d i$ is the fraction of wire with critical current in the interval di. For a Gaussian distribution

$$
P(i)=\frac{1}{\sigma \sqrt{2 \pi}} e^{-\left[\left(i-\bar{I}_{C}\right)^{2} / 2 \sigma^{2}\right]}
$$

Hampshire and Jones ${ }^{6}$ substituted Eq. (2) for $P(i)$ into Eq. (1) and extended the lower limit of integration to $-\infty$ to include the negative tail. The result is their Eq. (6) which predicts a finite voltage in the absence of current for finite $R$, a consequence of the artificially large values of $(I-i)$ that result from letting $i \rightarrow-\infty$. We also use Eq. (2) for $P(i)$ in Eq. (1) to evaluate the contribution of the positive part of the critical current distribution $(0<i \leqslant I)$. To add the effect of the delta function at $i=0$, we write the current in excess of the critical current in Eq. (1) as the 
total current $I$ instead of $(I-i)$, and then integrate from $-\infty$ to 0 to give the delta function its proper size (Fig. 1). The result is

$$
\begin{aligned}
V(I)= & \frac{R}{\sigma \sqrt{2 \pi}}\left(\int_{0}^{I} e^{-\left[\left(i-\widetilde{I}_{C}\right)^{2} / 2 \sigma^{2}\right]}(I-i) d i\right. \\
& \left.+I \int_{-\infty}^{0} e^{-\left[\left(i-\bar{I}_{C}\right)^{2} / 2 \sigma^{2}\right]} d i\right) .
\end{aligned}
$$

We let $\Delta=I-\bar{I}_{C}$ and change the variable of integration to $z=i-\bar{I}_{C}$ to obtain

$$
\begin{aligned}
V(I)= & \frac{R}{\sigma \sqrt{2 \pi}}\left(\int_{-\bar{I}_{C}}^{\Delta} e^{-\left(z^{2} / 2 \sigma^{2}\right)}(\Delta-z) d z\right. \\
& \left.+I \int_{-\infty}^{-\bar{I}_{C}} e^{-\left(z^{2} / 2 \sigma^{2}\right)} d z\right),
\end{aligned}
$$

then change it again to $s=\left(i-\bar{I}_{C}\right) /(\sigma \sqrt{2})$ and obtain

$$
\begin{aligned}
& V(I)=\frac{R}{\sqrt{\pi}}\left(\Delta \int_{-\left(\bar{I}_{C} \sigma \sigma \sqrt{2}\right)}^{\Delta / \sigma \sqrt{2}} e^{-s^{2}} d s-\sigma \sqrt{2} \int_{-\left(\bar{I}_{C} / \sigma \sqrt{2}\right)}^{\Delta / \sigma \sqrt{2}} e^{-s^{2} s d s}\right. \\
& \left.+I \int_{-\infty}^{-\left(\bar{I}_{d \sigma \sqrt{2})}\right.} e^{-s^{2}} d s\right) \\
& =\frac{R}{\sqrt{\pi}}\left(\Delta \int_{0}^{\Delta / \sigma \sqrt{2}} e^{-s^{2}} d s-\Delta \int_{0}^{\left(\bar{I}_{\text {Co }} / \sqrt{2}\right)} e^{-s^{2}} d s\right. \\
& +I \int_{0}^{-\left(\bar{I}_{C \sigma} \sigma \sqrt{2}\right)} e^{-s^{2}} d s+I \int_{-\infty}^{0} e^{-s^{2}} d s \\
& \left.-\sigma \sqrt{2} \int_{-\left(\bar{I}^{\prime} \sigma \sqrt{2}\right)}^{\Delta / \sigma \sqrt{2}} e^{-s^{2}} s d s\right) \\
& =\frac{R}{2}\left[\Delta \operatorname{erf}\left(\frac{\Delta}{\sigma \sqrt{2}}\right)+\bar{I}_{C} \operatorname{erf}\left(\frac{-\bar{I}_{C}}{\sigma \sqrt{2}}\right)+I\right. \\
& \left.=\frac{2 \sigma \sqrt{2}}{\sqrt{\pi}} \int_{-\left(\bar{I}_{C} \sigma \sqrt{2}\right)}^{\Delta \sigma \sqrt{2}} e^{-s^{2}} s d s\right]
\end{aligned}
$$

Changing variables once more to $u=s^{2}=\left(i-\bar{I}_{C}\right)^{2} /\left(2 \sigma^{2}\right)$ yields

$$
\begin{aligned}
V(I)= & \frac{R}{2}\left[\Delta \operatorname{erf}\left(\frac{\Delta}{\sigma \sqrt{2}}\right)+\bar{I}_{C} \operatorname{erf}\left(\frac{-\bar{I}_{C}}{\sigma \sqrt{2}}\right)+I\right. \\
& \left.-\frac{\sigma \sqrt{2}}{\sqrt{\pi}} \int_{\bar{I}_{c}^{2} / 2 \sigma^{2}}^{\Delta^{2} / 2 \sigma^{2}} e^{-u} d u\right] \\
= & \frac{R}{2}\left[\Delta \operatorname{erf}\left(\frac{\Delta}{\sigma \sqrt{2}}\right)-\bar{I}_{C} \operatorname{erf}\left(\frac{\bar{I}_{C}}{\sigma \sqrt{2}}\right)+I\right. \\
& \left.+\frac{\sigma \sqrt{2}}{\sqrt{\pi}}\left(e^{-\left(\Delta^{2} / 2 \sigma^{2}\right)}-e^{-\left(\bar{I}_{c}^{2} / 2 \sigma^{2}\right)}\right)\right]
\end{aligned}
$$

This analytic function is valid for any $I$ and shows proper limiting behavior. It is apparent that $V(0)=0$ and $V(I \rightarrow \infty)=I R$, and straightforward to show that $d V\left(\bar{I}_{C}\right) / d I=R / 2$ and $d V(I \rightarrow \infty) / d I=R$. The expression involves three unknown parameters: $R, \bar{I}_{C}$ and $\sigma$, and re- sembles the $V(I)$ expression proposed for a homogeneous superconductor with a Gaussian flux pinning force distribution. ${ }^{11}$

\section{EXPERIMENTAL RESULTS}

To test this new expression, we have compared its predictions to measurements on two rather different monofilament superconductors, shown in Fig. 2. We chose monofilaments in order to avoid the complications that may arise from sympathetic sausaging of multifilaments, as discussed by Warnes. ${ }^{4}$ In the first case we chose a very uniform, very high- $J_{C} \mathrm{Cu}$-clad $\mathrm{Nb}$-Ti monofilament. ${ }^{12} \mathrm{We}$ expect that the variation of both the local $J_{C}$ and the crosssectional area of the filament is very small, comparable to the very minimum that it is possible to achieve in a high $\kappa$ superconductor. The $\mathrm{Nb}-\mathrm{Ti}$ filament diameter is $46 \mu \mathrm{m}$, and the $\mathrm{Cu}$ cladding is $41 \mu \mathrm{m}$ thick. The largest filament irregularities visible in transverse cross section under a transmission electron microscope were $0.1 \mu \mathrm{m}$ across. The $60-\mathrm{cm}$-long sample was soldered in a spiral around the outside of a stainless-steel tube and measured over $33 \mathrm{~cm}$ in liquid $\mathrm{He}$ with fields up to $80 \%$ of $H_{C_{2}}$ applied along the axis of the spiral. Figure 3 compares the measured $V-I$ characteristics to Eq. (3); the fit is excellent. Table I displays values of the three adjustable parameters at each field. The small and nearly constant $\sigma / \bar{I}_{C}$ ratio $(0.09$ \pm 0.01 ) confirms the high uniformity of the wire.

Figure 4 shows the $V-I$ characteristics of a $3-\mathrm{cm}-$ long Ag-clad 2223 tape measured over $5 \mathrm{~mm}$ while immersed in liquid $\mathrm{N}_{2}$. The tape was prepared by the oxide-powder-intube method, as described clsewherc. ${ }^{13}$ It was rolled and then sintered three times with pressings between the heat treatments. It has a 2223 core $40 \mu \mathrm{m}$ thick and $2 \mathrm{~mm}$ wide, surrounded by a $40-\mu \mathrm{m}$-thick Ag sheath. Fields up to 200 $\mathrm{mT}$ were applied perpendicular to the plane of the tape, approximately parallel to the $2223 c$ axis. Figure 2 shows

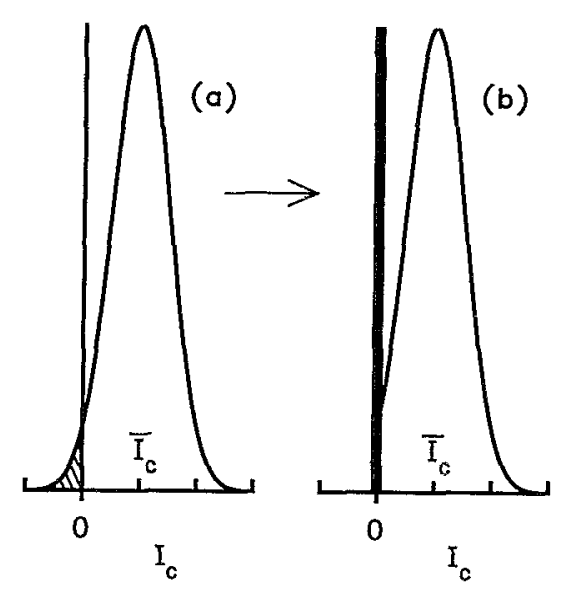

FIG. 1. If many variables affected $I_{C}$ independently, the central limit theorem predicts that $I_{C}$ would have a Gaussian distribution as in (a); however, all variables are similarly constrained from suppressing $I_{C}$ below zero, so the various effects are not completely independent. The $I_{C}$ distribution is thus truncated at zero as in (b). The area of the $\delta$ function at $I_{C}=0$ in (b) equals the area of the hatched negative region in (a). 

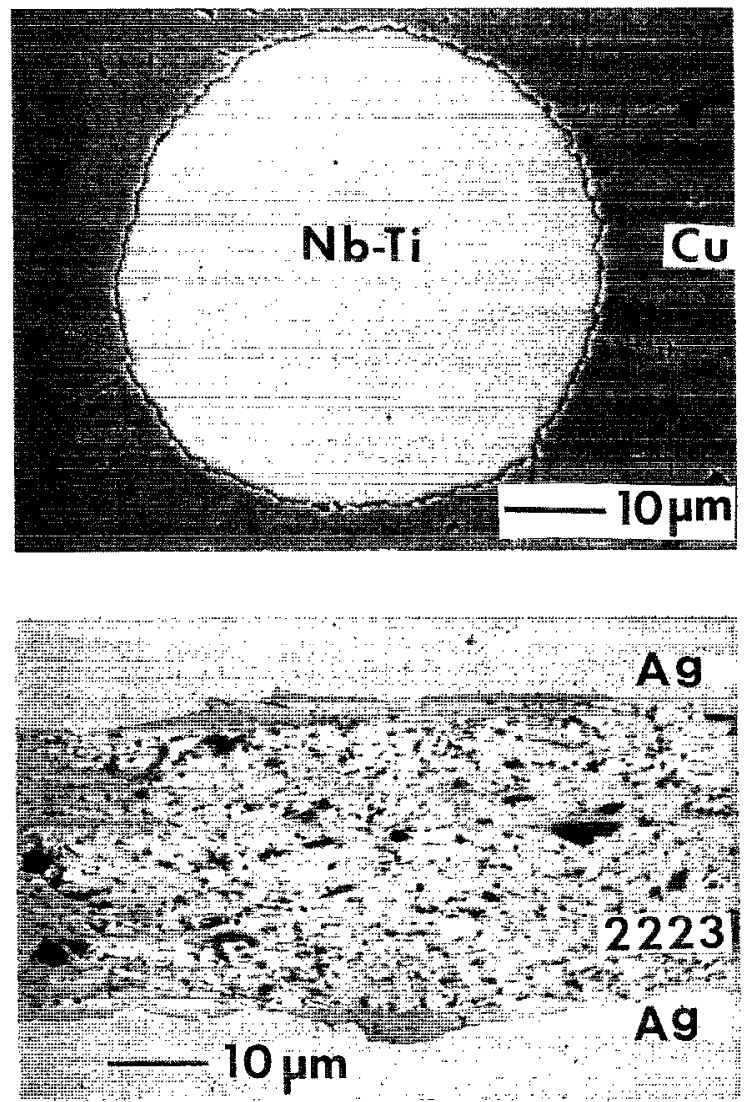

FIG. 2. These scanning electron micrographs show the two samples in cross section transverse to the direction of current flow. The upper photo shows the homogeneous $\mathrm{Cu}$-clad $\mathrm{Nb}$-Ti monofilament. The lower one shows dark second-phase regions dispersed throughout the Ag-sheathed 2223 tape.

that the microstructure is extremely variable from place to place, the scale of the irregularity being about $10 \mu \mathrm{m}$; however, the fit between the model and the data is excellent at all fields. In contrast to the $\mathrm{Nb}$ - Ti data, the $\sigma / I_{C}$ ratio is strongly variable, starting at 0.09 (the $\mathrm{Nb}-\mathrm{Ti}$ value) in zero

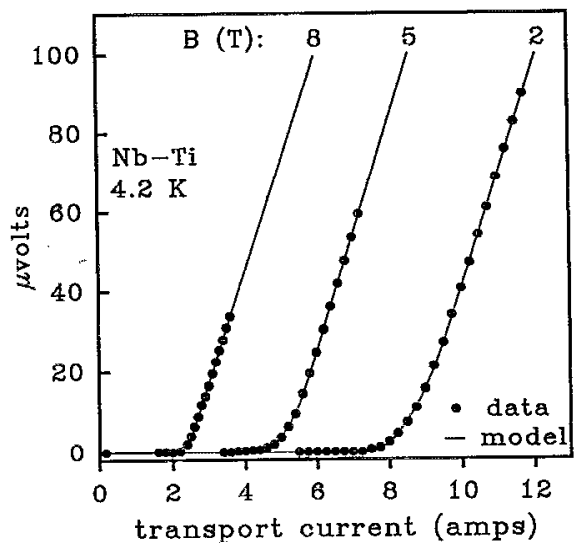

FIG. 3. $V-I$ curves for a $\mathrm{Nb}-\mathrm{Ti}$ monofilament in liquid $\mathrm{He}$ at three applied fields. The lines represent Eq. (3) with parameter values from Table I.
TABLE I. Mean and standard deviation of the critical current and their quotient, and shunt resistance $R$ from fitting Eq. (3). Independent measurements of $R$ are also shown.

\begin{tabular}{llccccc}
\hline \hline Sample & $\begin{array}{c}\text { Field } \\
(\mathrm{T})\end{array}$ & $\begin{array}{c}\bar{I}_{C} \\
(\mathrm{~A})\end{array}$ & $\begin{array}{c}\sigma \\
(\mathrm{A})\end{array}$ & $\begin{array}{c}\sigma / \bar{I}_{C} \\
(\%)\end{array}$ & $\begin{array}{c}R \text { fit } \\
(\mu \Omega)\end{array}$ & $\begin{array}{c}R \\
\text { measured } \\
(\mu \Omega)\end{array}$ \\
\hline Nb-Ti & 2 & 8.58 & 0.75 & 8.7 & 28 & \\
mono- & & & & & & \\
filament & 5 & 5.15 & 0.49 & 9.6 & 29 & $41 \pm 8$ \\
at 4.2 K & 8 & 2.38 & 0.18 & 7.8 & 28 & \\
2223 & 0 & 5.77 & 0.53 & 9.2 & 57 & \\
tape & 0.005 & 3.69 & 0.57 & 15.6 & 56 & \\
at 77 K & 0.01 & 2.31 & 0.45 & 19.4 & 58 & $70 \pm 2$ \\
& 0.02 & 1.72 & 0.44 & 25.8 & 61 & \\
& 0.05 & 1.11 & 0.28 & 25.6 & 60 & \\
& 0.2 & 0.56 & 0.26 & 46.9 & 63 & \\
\hline \hline
\end{tabular}

field, and then rapidly increasing to a maximum of 0.47 in a field of only $200 \mathrm{mT}$. Although $H_{C_{2}}$ is uncertain for such tapes, the applied fields were definitely less than $0.1 H_{C_{2}}$. This aspect of the $V-I$ data contrasts with that observed for the $\mathrm{Nb}$-Ti sample.

We deliberately chose two very different samples. The particular differences that we thought would be significant are the presence of giant flux creep in the 2223 tape $^{14}$ and its absence in the $\mathrm{Nb}-\mathrm{Ti}$, and the uniform microstructure of the Nb-Ti compared to the highly inhomogeneous microstructure of the 2223 . The model fits the data equally well in both cascs. Table $I$ includes independent estimates of the shunt resistance $R$, obtained by measuring the parallel resistance of the steel tube, solder and $\mathrm{Cu}$ cladding on the $\mathrm{Nb}-\mathrm{Ti},{ }^{15}$ and by measuring the $\mathrm{Ag}$ sheath on the 2223. Virtually all current in excess of $I_{C}$ at a particular cross section is shunted into the normal metal. ${ }^{9}$ For both samples the model gives values of the asymptotic slope $R$ close to, but slightly below, the independent estimates. The model values may be too low if the data do not include sufficiently high currents. The values of $\sigma / I_{C}$ for $\mathrm{Nb}-\mathrm{Ti}$ are consistent with detailed measurements on a wide range of composites, for which the $n$ values have been correlated to

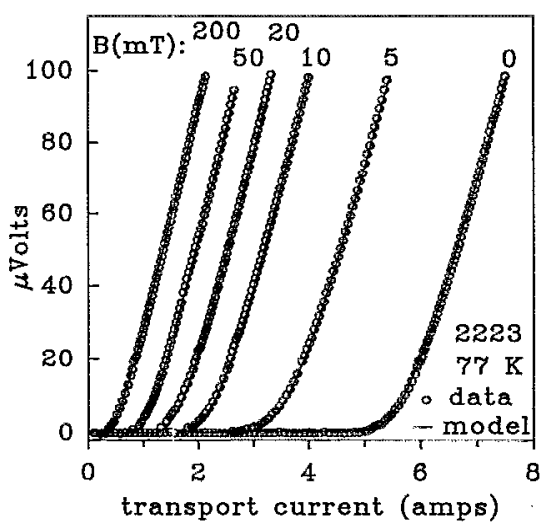

FIG. 4. $V-I$ curves for a 2223 tape in liquid $\mathrm{N}_{2}$ at six applied fields. The lines represent Eq. (3) with parameter values from Table I. 
the distribution of filament cross sections in multifilamentary composites. 316

\section{ORIGIN OF THE $\boldsymbol{N}$-VALUE}

A substantial portion of most $V-I$ characteristics can be approximated by the expression ${ }^{17,18}$

$$
V \propto I^{n}
$$

where the exponent $n$ is given by

$$
n=\frac{d[\log (V)]}{d[\log (I)]}=\frac{d[\log (V)]}{d V} \frac{d V}{d I} \frac{d I}{d[\log (I)]}=\frac{I}{V} \frac{d V}{d I} .
$$

We can differentiate the analytic expression for $V(I)$, Eq. (3), to evaluate $n$. The result is

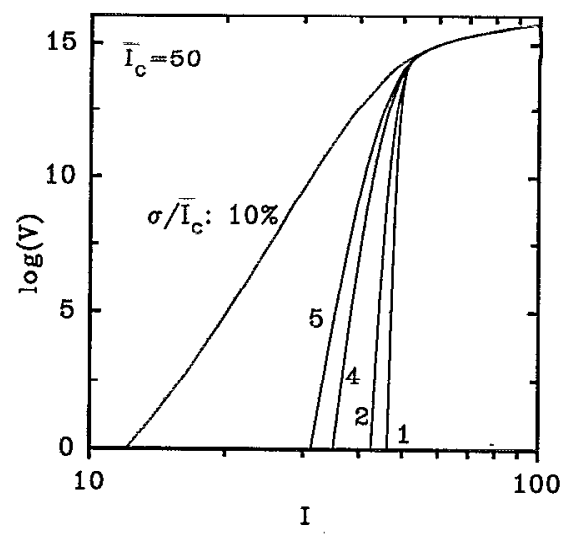

FIG. 5. Equation (3) over 16 decades of $V$ using several ratios of standard deviation to mean critical current. Equation (4) gives the slope or $n$ value at any point. Units are arbitrary.

$$
n=\frac{I \operatorname{erf}(\Delta / \sigma \sqrt{2})+I}{\Delta \operatorname{erf}(\Delta / \sigma \sqrt{2})-\bar{I}_{C} \operatorname{erf}\left(\bar{I}_{C} / \sigma \sqrt{2}\right)+I+\sigma \sqrt{2} / \sqrt{\pi}\left(e^{-\left(\Delta^{2} / 2 \sigma^{2}\right)}-e^{-\left(\bar{I}_{c}^{2} / 2 \sigma^{2}\right)}\right)} .
$$

Figure 5 shows a $\log (V)-\log (I)$ plot of Eq. (3) for several $\sigma / \bar{I}_{C}$ values. The slope or " $n$ value" of these curves at any point is given by Eq. (4). While the characteristics all have finite curvature at finite $V$, for $I<\bar{I}_{C}$ the curvature is barely perceptible and $n$ is reasonably well defined. However, $n$ generally does increase with decreasing electric field, so that measured $n$ values should be at least a weak function of voltage sensitivity.

In summary, we have derived an exact expression for $V(I)$ that is valid for any $I$ in a sample with a Gaussian critical-current distribution. This expression provides excellent fits to data from two very different types of highfield superconductor, a Cu-clad Nb-Ti monofilament and a $\mathrm{Bi}_{2} \mathrm{Sr}_{2} \mathrm{Ca}_{2} \mathrm{Cu}_{3} \mathrm{O}_{10} \mathrm{Ag}$-sheathed tape. We have further derived an exact expression for the heretofore empirical $n$ value, and we have also illustrated its weak voltage dependence.

\section{ACKNOWLEDGMENTS}

We wish to thank Lance Cooley for the Nb-Ti data. The work has been supported by Electric Power Research Institute (Contract No. RP 8009-05) and the Advanced
Research Projects Agency (Contract No. N00014-90-J4115).

${ }^{1}$ R. G. Jones, E. H. Rhoderick, and A. C. Rose-Innes. Phys. Lett. A 24, 318 (1967).

${ }^{2}$ J. Baixeras and G. Fournet, J. Phys. Chem. Solids 28, 1541 (1967).

${ }^{3}$ W. H. Warnes and D. C. Larbalestier, Cryogenics 26, 643 (1986).

${ }^{4}$ W. H. Warnes, J. Appl. Phys. 63, 1651 (1988).

${ }^{5}$ C. J. G. Plummer and J. E. Evetts, IEEE Trans. Magn. MAG-23, 1179 (1987).

${ }^{6}$ D. P. Hampshire and H. Jones, Cryogenics 27, 608 (1987).

${ }^{7}$ M. Tinkham, Phys. Rev. Lett. 61, 1658 (1988).

${ }^{8}$ R. J. Soulen, Jr., IEEE Trans. Appl. Supercond. 3, 1261 (1993).

${ }^{9}$ A. Gurevich, A. E. Pashitski, H. S. Edelman, and D. C. Larbalestier, Appl. Phys. Lett. 62, 1688 (1993).

${ }^{10} \mathrm{~J}$. E. Evetts, B. A. Glowacki, P. L. Sampson, and M. G. Blamire, IEEE Trans. Magn. MAG-25, 2041 (1989).

${ }^{11}$ O. V. Magradze, L. V. Matyushkina, and V. A. Shukhman, J. LowTemp. Phys. 55, 475 (1984).

${ }^{12}$ C. Meingast and D. C. Larbalestier, J. Appl. Phys. 49, 5971 (1989).

${ }^{13}$ A. Umezawa, Y. Feng, H. S. Edelman, Y. E. High, D. C. Larbalestier, Y. S. Sung, and E. E. Hellstrom, Physica C 198, 261 (1992).

${ }^{14}$ Y. Yeshurun and A. P. Malozemoff, Phys. Rev. Lett. 60, 2202 (1988).

${ }^{15}$ W. H. Warnes, Ph.D. thesis, University of Wisconsin-Madison, 1986, p. 90.

${ }^{16}$ Y. E. High, P. J. Lee, J. C. McKinnell, and D. C. Larbalestier, Adv. Cryog. Eng. 36, 647 (1992).

${ }^{17}$ F. Volker, Part. Accel. 1, 205 (1970)

${ }^{18}$ J. Ekin, J. Appl. Phys. 49, 3406 (1978). 